\section{Covid-19: EMA defends AstraZeneca vaccine as Germany and Canada halt rollouts}

\author{
Owen Dyer
}

The Oxford AstraZeneca covid-19 vaccine is facing a further wave of scepticism and restrictions as Canada and Germany halt its use in younger patients, citing reports of clotting disorders in recently vaccinated people.

On 30 March, Canadian provinces suspended the use of the vaccine in people aged under 55 . The pause was recommended by the country's National Advisory Committee on Immunization (NACI), which pointed to reports of "rare cases of serious blood clots" in Europe. ${ }^{1}$ These included instances of cerebral venous sinus thrombosis (CVST), the agency said. Most but not all cases had occurred in women aged under 55 , and the syndrome typically appeared 4 to 16 days after receiving the vaccine. The fatality rate among those developing such clots had been as high as $40 \%$, said Shelley Deeks, vice chair of NACI, announcing the recommendations. "There is substantial uncertainty about the benefit of providing AstraZeneca covid-19 vaccines to adults under 55, given the potential risks," she said, presenting the recommendation as a temporary measure while more data are gathered.

The announcement came just as Canada expected delivery of 1.5 million doses of the AstraZeneca vaccine offered by US president Joe Biden. About 500 ooo shots of the vaccine have been given in Canada so far.

Several Canadian newspapers accused the government of overreacting just as the country slides into a third wave. "Constantly changing guidelines are undermining public trust in all vaccines," argued the editorial board of the Toronto Star, noting that NACI had once recommended against the AstraZeneca vaccine in the over-65s. ${ }^{2}$

\section{Germany looks towards Sputnik}

On 31 March, German states suspended the use of the AstraZeneca vaccine in the under-6os, following a draft recommendation from Germany's Standing Immunisation Committee (STIKO). ${ }^{3}$ Unlike Canada's recommendation, STIKO does not counsel a temporary pause while clots are investigated, but a lasting halt.

STIKO has not decided what course to recommend for people under 60 who have already had one AstraZeneca shot, but will soon announce a plan, it said. Meanwhile Germany's chancellor Angela Merkel this week joined French president Emmanuel Macron in a phone call with Russia president Vladimir Putin to discuss potentially obtaining the Sputnik vaccine.

Germany's decision leaves the AstraZeneca vaccine stopped in four European countries-Denmark, Latvia, the Netherlands, and Norway-and age restricted in six more-Finland, France, Germany, Iceland, Lithuania, and Sweden. It is used for all adults in 12 European countries: Austria, Bulgaria, Cyprus, Estonia, Ireland, Italy, Luxembourg, Portugal, Romania, Slovenia, Spain, and the UK.

Neither AstraZeneca nor the vaccine's developers at Oxford University commented on the German and Canadian decisions. But the European Medicines Agency (EMA) came to the vaccine's defence, arguing that the number of clotting events described by German authorities was out of line with global experience. Germany has reported 31 cases of cerebral thrombosis in 2.7 million doses of the AstraZeneca vaccine, roughly one incident per 90 ooo shots.

The EMA is investigating 62 case reports of CVST from around the world, including 44 total cases from the European Economic Area, in which 9.2 million shots of the vaccine have been given, suggesting one incident per 210000 shots. Not all the German reported cases are in the EMA's data, however.

EMA chief Emer Cooke said there is no proof of any causal link between the vaccine and the clotting disorder, a syndrome of which frequency in normal years has never been reliably measured. Cooke released the preliminary EMA report into the matter, which concludes that "the benefits of the AstraZeneca covid vaccine, with the latest data suggesting an $85 \%$ reduction in hospital admissions and death from covid-19, far outweigh any possible risks." 4

\section{Clotting theory explained}

The EMA's report and the new Canadian guidance described the mechanism postulated by researchers who do believe that the vaccine is triggering clots. Unusually, patients exhibit thrombocytopenia, a shortage of clotting platelets in blood tests, even as they suffer clots. The same apparent contradiction is seen in heparin induced thrombocytopenia, and also sometimes in thrombosis associated with covid-19.

This type of adverse event is being named vaccine induced prothrombotic immune thrombocytopenia. An antigen or protein generated by vaccination with AstraZeneca's product is activating platelets to begin clotting, University of Greifswald researcher Andreas Greinacher told a press conference on 31 March. His team's research awaits publication in the Lancet but a draft version, not peer reviewed, is online. ${ }^{5}$

Greinacher expressed astonishment that their vaccine. "People being severely sickened by covid-19 outnumber those who suffer from the vaccination reaction by several orders of magnitude," he said. "To stop or to avoid vaccination only for the fear of findings were being used to justify restricting the 
getting an extremely rare, adverse reaction would be completely wrong."

But such reassurance is increasingly falling on deaf ears in countries where the vaccine is a distant third favourite behind those of Moderna and Pfizer. In Ontario, despite soaring covid-19 case numbers, premier Doug Ford said this week, "I'd rather wait a month or two for Pfizer, Moderna, and Johnson \& Johnson than roll the dice on AstraZeneca.”

Bavaria’s prime minister Markus Söder complained that repeated bad news stories had sunk in with the public. "At AstraZeneca we can expect some new problem every day. And you can feel that in the perception outside," he said, arguing that the vaccine should simply be given to "whoever wants it and who dares to do it."

1 Health Canada. NACl rapid response: recommended use of AstraZeneca covid-19 vaccine in younger adults. 30 March 2021. www.canada.ca/en/public-health/services/immunization/nationaladvisory-committee-on-immunization-naci/rapid-response-recommended-use-astrazenecacovid-19-vaccine-younger-adults.html.

2 Star editorial board. Constantly changing guidelines are undermining public trust in all vaccines. 29 March 2021. www.thestar.com/opinion/editorials/2021/03/29/constantly-changing-guidelinesare-undermining-public-trust-in-all-vaccines.html.

3 STIKO press release from 30 March 2021. 2021. www.rki.de/DE/Content/Kommissionen/STIKO/Empfehlungen/AstraZeneca-Impfstoff-2021-03-30.html.

4 European Medicines Agency. Signal assessment report on embolic and thrombotic events with covid-19 vaccine (ChAd0x1-S [recombinant]): Covid-19 vaccine AstraZeneca (other viral vaccines) 24 March 2021. www.ema.europa.eu/en/documents/prac-recommendation/signal-assessmentreport-embolic-thrombotic-events-smq-covid-19-vaccine-chadox1-s-recombinant-covid_en.pdf.

5 Greinacher A, Thiele T, Warkentin TE, et al. A prothrombotic thrombocytopenic disorder resembling heparin-induced thrombocytopenia following coronavirus-19 vaccination. Research Square. 29 March 2021. www.researchsquare.com/article/rs-362354/v1. 